\title{
Rupture of an infected radial artery false aneurysm
}

\author{
Kamal Hachem, MD · Jihane Kfoury, MD · Joanna Tohmé, MD • Viviane Chalhoub, MD
}

Received: 17 August 2016/Revised: 23 August 2016/Accepted: 1 September 2016/Published online: 1 November 2016 (C) Canadian Anesthesiologists' Society 2016

A 67-yr-old male obese smoker was admitted to the intensive care unit with respiratory failure due to exacerbation of chronic obstructive pulmonary disease. A 20G, 5-cm catheter (Seldicath; Plastimed ${ }^{\circledR}$, Paris, France) was placed in the right radial artery under aseptic conditions with a single puncture. The catheter was removed on day 5 and its tip sent for routine culture. On day 7 , a red, painful, pulsatile mass (Figure A) with purulent discharge appeared at the puncture site. The discharge was sent for culture. Arterial ultrasonography of the lesion revealed a radial artery pseudoaneurysm (Figures B and C). The cultures from both the catheter tip and discharge were positive for methicillin-resistant Staphylococcus aureus. Antibiotic treatment was initiated with teicoplanine on day 9. On day 11, active bleeding at the puncture site developed. A suspected rupture of the
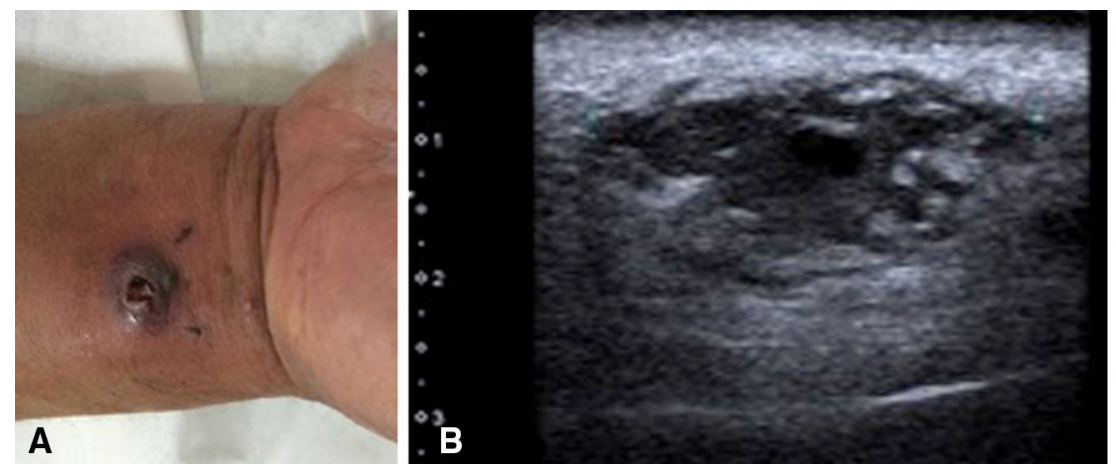

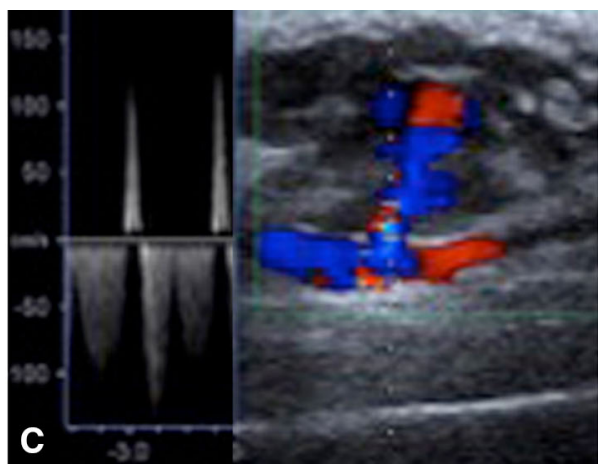

imaging (panel C) reveals the 8-mm saccular pseudoaneurysm containing circulating blood communicating with the lumen of the radial artery. Pulsed-wave Doppler imaging detects the to-and-fro blood flow through the neck of the pseudoaneurysm

K. Hachem, MD (凶) · J. Kfoury, MD

Department of Radiology, Saint Joseph University Medical

School, Hotel Dieu de France Hospital, Beirut, Lebanon

e-mail: hachemkam@hotmail.com

J. Tohmé, MD · V. Chalhoub, MD

Department of Anesthesiology and Intensive Care, Saint Joseph

University Medical School, Hotel Dieu de France Hospital,

Beirut, Lebanon 
pseudoaneurysm was first controlled by manual compression until transfer to the operating room, where the radial artery was ligated and the infected false aneurysm excised. The patient was discharged home three days later.

Infected radial artery pseudoaneurysm is a rare but serious complication of arterial catheterization and may occur days or weeks following catheter removal. Local infection is the most common risk factor for pseudoaneurysm. ${ }^{1}$ Strong predictors of a false aneurysm are Staphylococcus aureus infection and persistence of bacteremia for more than 48 hours following catheter removal and antibiotic therapy. Staphylococcus aureus has been isolated in more than $90 \%$ of all reported cases. A ruptured false aneurysm can be serious and may occur days or weeks following discharge from the hospital. Surgical treatment of an infected radial artery pseudoaneurysm, including excision of the false aneurysm and repair or ligation of the artery, is strongly indicated.

Conflicts of interest None declared.

Editorial responsibility This submission was handled by Dr. Hilary P. Grocott, Editor-in-Chief, Canadian Journal of Anesthesia.

Funding source No funding source.

\section{Reference}

1. Garg K, Howell BW, Saltzberg SS, et al. Open surgical management of complications from indwelling radial artery catheters. J Vasc Surg 2013; 58: 1325-30. 